\title{
A novel missense PTEN mutation identified in a patient with macrocephaly and developmental delay
}

\author{
Yuichi Ueno ${ }^{1}$, Takashi Enokizono ${ }^{1}$, Hiroko Fukushima ${ }^{1,2}$, Tatsuyuki Ohto $\mathbb{1}^{1,2}$, Kazuo Imagawa ${ }^{1}$, Mai Tanaka ${ }^{1}$, Aiko Sakai ${ }^{1}$,
} Hisato Suzuki $\mathbb{0}^{3}$, Tomoko Uehara ${ }^{3}$, Toshiki Takenouchi ${ }^{3}$, Kenjiro Kosaki ${ }^{3}$ and Hidetoshi Takada ${ }^{1,2}$

\begin{abstract}
Phosphatase and tensin homolog (PTEN) plays an important role in tumor suppression. A germline mutation in the PTEN gene induces not only PTEN hamartoma tumor syndrome, including Cowden syndrome, but also macrocephaly/ autism syndrome. Here, we describe a boy with macrocephaly/autism syndrome harboring a novel missense heterozygous PTEN mutation, c.959T>C (p.Leu320Ser). Interestingly, a previously reported nonsense mutation resulting in p.Leu320X was found in Cowden syndrome patients. Our case may be suggestive of a genotype-phenotype correlation.
\end{abstract}

The phosphatase and tensin homolog (PTEN) gene (OMIM 601728), located on chromosome 10q23, is a tumor suppressor gene that has a significant role in cellular proliferation, migration, and apoptosis ${ }^{1}$. PTEN hamartoma tumor syndrome (PHTS), which comprises Cowden syndrome (CS; OMIM 158350), Bannayan-Riley-Ruvalcaba syndrome (BRRS; OMIM 153480), and Proteus-like syndrome, represents a spectrum of hamartomatous overgrowth manifestations associated with germline mutations in the PTEN gene ${ }^{2}$. CS is characterized by macrocephaly, trichilemmomas, facial acral keratosis, hamartoma, and an increased risk of certain cancers, particularly of the breast, uterus, and thyroid ${ }^{3}$. Under revised diagnostic criteria, the majority of CS diagnoses are made in adulthood because cutaneous findings or malignancies are rarely present before 20 years of age $\mathrm{e}^{4}$. BRRS is typically diagnosed in childhood and is characterized by macrocephaly, hamartomas (including lipomas or intestinal polyps), penile freckling in males and developmental delays, including an increased risk

\footnotetext{
Correspondence: Takashi Enokizono (tenokizono-tkb@umin.ac.jp) ${ }^{1}$ Department of Pediatrics, University of Tsukuba Hospital, Tsukuba, Japan ${ }^{2}$ Department of Child Health, Faculty of Medicine, University of Tsukuba, Tsukuba, Japan

Full list of author information is available at the end of the article.
}

of autism spectrum disorder (ASD). Because the clinical presentation of CS and BRRS is quite similar, they were considered to be a single disorder with variable phenotypic expression and age-related penetrance ${ }^{5}$. However, the clinical features of patients with PTEN mutations were quite variable, and presentations differed significantly even within the same family ${ }^{6}$.

PTEN mutations have been identified in children with macrocephaly associated with ASD and/or developmental delay without hamartomas known as macrocephaly/autism syndrome (OMIM 605309) $)^{7,8}$. However, the clinical features of these children have not been described in detail. We report here a 4-year-old boy with macrocephaly and developmental delay that had a novel missense mutation, NM_000314.4:c.959T>C [p.(Leu320Ser)], in the PTEN gene.

The patient was the first child of healthy nonconsanguineous Japanese parents. Family history was unremarkable. He was born after 38 weeks of gestation using vacuum extraction with a birth weight of $3.696 \mathrm{~g}$ $(+1.7 \mathrm{SD})$, length of $52 \mathrm{~cm}(+1.4 \mathrm{SD})$ and head circumference of $37 \mathrm{~cm}(+2.6 \mathrm{SD})$. He could control his head at 5 months but was referred to our hospital for

\section{(c) The Author(s) 2019}

(c) (i) Open Access This article is licensed under a Creative Commons Attribution 4.0 International License, which permits use, sharing, adaptation, distribution and reproduction cc) in any medium or format, as long as you give appropriate credit to the original author(s) and the source, provide a link to the Creative Commons license, and indicate if changes were made. The images or other third party material in this article are included in the article's Creative Commons license, unless indicated otherwise in a credit line to the material. If material is not included in the article's Creative Commons license and your intended use is not permitted by statutory regulation or exceeds the permitted use, you will need to obtain permission directly from the copyright holder. To view a copy of this license, visit http://creativecommons.org/licenses/by/4.0/. 


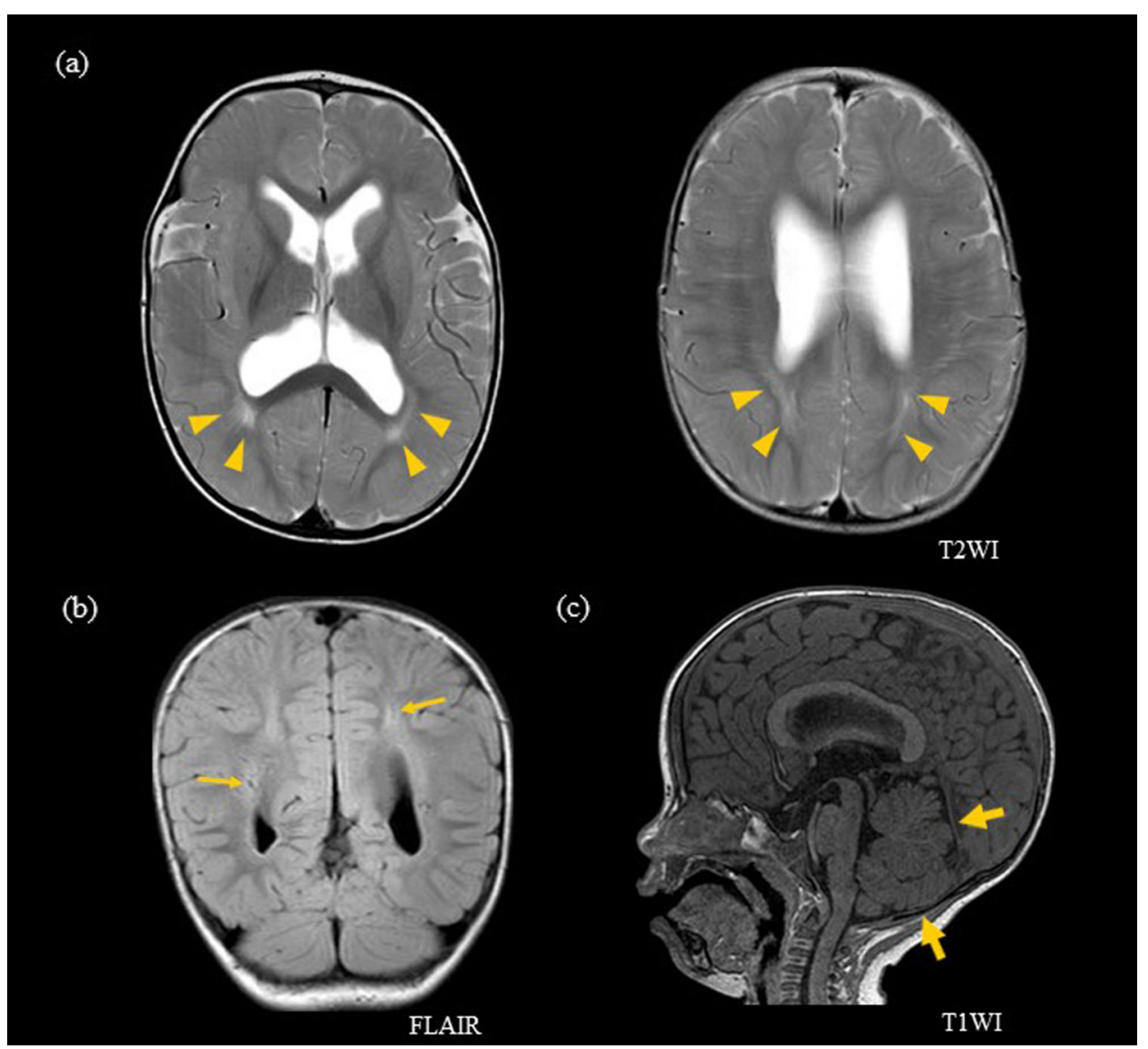

Fig. 1 Head MRI at 10 months of age. a Axial T2-weighted image and $\mathbf{b}$ coronal FLAIR image show hyperintensity in the bilateral periventricular white matter regions (yellow arrowhead) and enlarged periventricular spaces (thin yellow arrow). c Sagittal T1-weighted image shows a narrow posterior fossa (broad yellow arrow)

developmental delay and muscular hypotonia at 10 months of age.

His head circumference was $50.0 \mathrm{~cm}(+2.8 \mathrm{SD})$ at 10 months old. His anterior fontanelle was widely open with a prominent forehead, but he did not have any symptoms related to increased intracranial pressure, such as vomiting, seizures, or poor activity. He showed loose shoulder and heel to ear sign, suggesting general muscular hypotonus. Physical examination did not reveal heart murmur, organomegaly, cutaneous pigmentary lesions, or freckles on the penis. The overall developmental quotient was 60 by the Kinder Infant Development Scale ${ }^{9}$. Head MRI at 10 months showed white matter hyperintense areas on T2 weighted and fluid-attenuated inversion recovery (FLAIR) images, enlarged perivascular spaces, mild ventricular enlargement, and narrow posterior fossa (Fig. 1). The possibility of hydrocephalus was excluded after cisternography at 1 year and 1 month of age. He could walk alone at 1 year and 8 months of age. He spoke the first meaningful word at 1 year and 5 months and two phrases at 2 years and 6 months. The physical examination at 4 years and 2 months of age showed a weight of $17.4 \mathrm{~kg}(+0.7 \mathrm{SD})$, height of $104.0 \mathrm{~cm}(+0.7 \mathrm{SD})$, and head circumference of $56.5 \mathrm{~cm}(+3.6 \mathrm{SD})$. Chromosomal analysis showed $46, \mathrm{XY}$.

A trio exome analysis was performed as described previously $^{10}$. The research protocol was approved by the local Institutional Ethical Review Board of the University of Tsukuba and Keio University School of Medicine. Informed consent was obtained from the parents on the basis of the Declaration of Helsinki. Genomic DNA was extracted from peripheral blood leukocytes of the patient and his parents. Whole-exome sequencing using the SureSelectXT2 Human All Exon Kit V4 (Agilent Technologies, Santa Clara, CA) was performed on the HiSeq 1000 platform (Illumina, San Diego, CA) and showed a heterozygous missense mutation, NM_000314.4:c.959T $>C$, [p.(Leu320Ser)] in exon 8 of PTEN (OMIM \#601728) that was confirmed by Sanger sequencing (Fig. 2). This mutation was found only in the patient and not in his parents, indicating that it was a de novo mutation. In silico analysis demonstrated that this mutation was likely pathogenic 


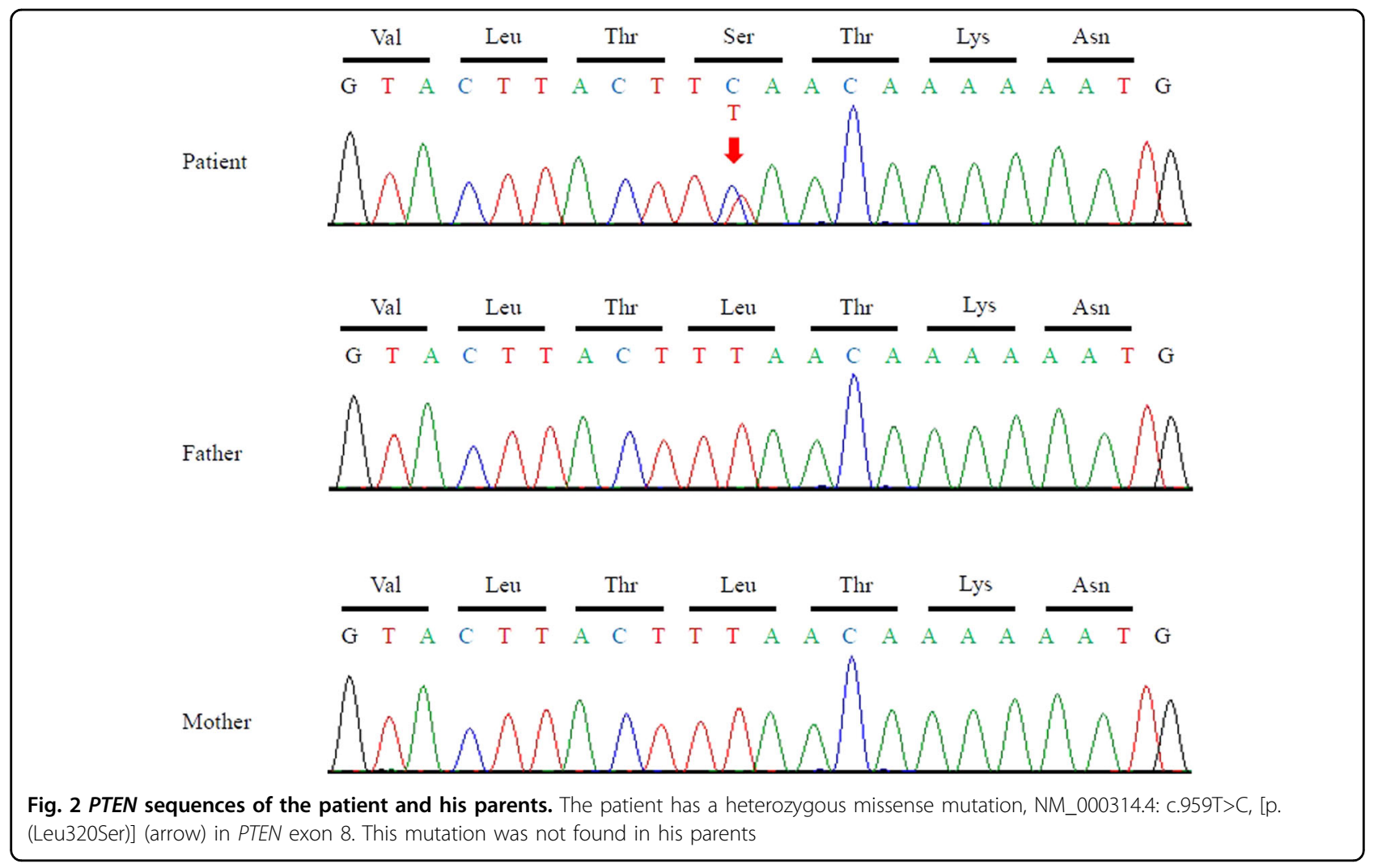

(SIFT: deleterious, score 0; PolyPhen: probably damaging, score 0.99; PROVEAN: damaging, score -4.2). To the best of our knowledge, this mutation has never been reported previously. Based on a literature review, the variants c. $959 \mathrm{~T}>\mathrm{A}$ and c. $959 \mathrm{~T}>\mathrm{G}$, which result in p.Leu320X, have been previously reported in CS patients ${ }^{4,11}$.

In 2005, Butler et al. ${ }^{8}$ reported the presence of heterozygous germline mutations in the PTEN gene in three children with ASD and macrocephaly. Since then, germline PTEN pathogenic variants have been reported in $10-20 \%$ of ASD cases with macrocephaly. Furthermore, developmental delay, intellectual disability, and/or ASD have been recorded in $66 \%$ of children with pathogenic mutations in $P T E N^{3}$.

Our patient presented with macrocephaly from birth, whereas developmental delay and muscular hypotonia without dermatological findings were noted from the infantile period. His brain MRI was consistent with previous reports of individuals with PTEN mutations who also presented with macrocephaly, developmental delay, and/or $\mathrm{ASD}^{4}$. Whole-exome sequencing identified a novel missense PTEN gene mutation, NM_000314.4:c.959T>C, [p.(Leu320Ser)], in the patient. However, he did not meet clinical criteria for BRRS or CS diagnoses. Although we diagnosed him with macrocephaly/autism syndrome provisionally, it is certainly possible that the patient will meet the criteria for CS/BRRS in the future.
Many of the mutations associated with CS and BRRS occur in exons 5,7 , and 8 of the PTEN gene ${ }^{11}$. Previous studies of CS/BRRS cohorts have failed to establish clear genotype-phenotype correlations ${ }^{3}$. Furthermore, Frazier et al. $^{12}$ reported that germline heterozygous missense PTEN mutations were enriched in ASD and macrocephaly cases compared to their frequency in PHTS patients without ASD. Interestingly, the missense mutation Leu320Ser found in our patient was associated with macrocephaly and developmental delay, whereas the previously reported nonsense mutation Leu320X was found in a CS patient. Our case may be suggestive of a genotype-phenotype correlation even though we could not confirm this on the basis of a single case report. Further research is needed to ascertain this correlation.

The major concern in patients with PTEN mutations is a risk of malignancy. To date, however, a consensus cancer surveillance protocol has not been formally instituted, so all PTEN mutation carriers should adopt the cancer surveillance strategies proposed for patients with $\mathrm{CS}^{2}$. We started annual general surveillance and annual specific surveillance of thyroid cancer for future cancer risk in this patient.

In conclusion, we identified a novel missense PTEN gene mutation in a Japanese boy who presented with developmental delay and macrocephaly. We will continue to follow up with this patient regularly for the early detection of tumor development as well as to expand our 


\section{knowledge of genotype-phenotype correlations in patients with pathological PTEN variants.}

\section{HGV Database}

The relevant data from this Data Report are hosted at the Human Genome Variation Database at https://doi.org/10.6084/m9.figshare.hgv.2579.

\section{Acknowledgements}

We would like to express our gratitude to the patient and his family.

\section{Funding}

This study was supported by the Initiative on Rare and Undiagnosed Diseases from the Japan Agency for Medical Research and Development and Research on Rare and Intractable Diseases from the Ministry of Health, Labor and Welfare, Japan.

\section{Author details}

${ }^{1}$ Department of Pediatrics, University of Tsukuba Hospital, Tsukuba, Japan. ${ }^{2}$ Department of Child Health, Faculty of Medicine, University of Tsukuba, Tsukuba, Japan. ${ }^{3}$ Center for Medical Genetics, Keio University School of Medicine, Tokyo, Japan

\section{Conflict of interest}

The authors declare that they have no conflict of interest.

\section{Publisher's note}

Springer Nature remains neutral with regard to jurisdictional claims in published maps and institutional affiliations.

Received: 15 February 2019 Revised: 6 April 2019 Accepted: 8 April 2019. Published online: 23 May 2019

\section{References}

1. Sansal, I. \& Sellers, W. R. The biology and clinical relevance of the PTEN tumor suppressor pathway. J. Clin. Oncol. 22, 2954-2963 (2004).

2. Piccione, $M$. et al. PTEN hamartoma tumor syndromes in childhood: description of two cases and a proposal for follow-up protocol. Am. J. Med. Genet. A. 161A, 2902-2908 (2013).

3. Hansen-Kiss, E. et al. A retrospective chart review of the features of PTEN hamartoma tumour syndrome in children. J. Med. Genet. 54, 471-478 (2017).

4. Vanderver, A. et al. Characteristic brain magnetic resonance imaging pattern in patients with macrocephaly and PTEN mutations. Am. J. Med. Genet. A. 164A 627-633 (2014).

5. Marsh, D. J. et al. PTEN mutation spectrum and genotype-phenotype correlations in Bannayan-Riley-Ruvalcaba syndrome suggest a single entity with Cowden syndrome. Hum. Mol. Genet. 8, 1461-1472 (1999).

6. Varga, E. A., Pastore, M., Prior, T., Herman, G. E. \& McBride, K. L. The prevalence of PTEN mutations in a clinical pediatric cohort with autism spectrum disorders, developmental delay, and macrocephaly. Genet. Med. 11, 111-117 (2009).

7. Herman, G. E. et al. Increasing knowledge of PTEN germline mutations: Two additional patients with autism and macrocephaly. Am. J. Med. Genet. Part. A. 143A, 589-593 (2007).

8. Butler, M. G. et al. Subset of individuals with autism spectrum disorders and extreme macrocephaly associated with germline PTEN tumour suppressor gene mutations. J. Med. Genet. 42, 318-321 (2005).

9. Miyake, K., Ohmura, M., Takashima, M., Yamauchi, S. \& Hashimoto, Y. Kinder Infant Development Scale: Manual. (Hattatsu Kagaku Kenkyu Kyoiku Center, Tokyo, 1989). [in Japanese].

10. Takenouchi, T. et al. Novel overgrowth syndrome phenotype due to recurrent de novo PDGFRB mutation. J. Pediatr. 166, 483-486 (2015).

11. Eng, C. PTEN: one gene, many syndromes. Hum. Mutat. 22, 183-198 (2003).

12. Frazier, T. W. et al. Molecular and phenotypic abnormalities in individuals with germline heterozygous PTEN mutations and autism. Mol. Psychiatry. 20, 1132-1138 (2014) 